\title{
Context Enabled Query and Minimalist Metadata Visualization: A Context Bound Approach for User and Content
}

\author{
Fausto Giunchiglia \\ Department of Computer \\ Science \\ University of Trento, \\ Trento, Italy
}

\author{
Khandaker Tabin \\ Hasan \\ Department of Computer \\ Science \\ University of Trento, \\ Trento, Italy
}

\author{
Rezwan Ahmed \\ Department of Computer \\ Science \\ American International \\ University-Bangladesh, \\ Dhaka, Bangladesh
}

\author{
Sheikh Shaugat \\ Abdullah \\ Department of Computer \\ Science \\ American International \\ University-Bangladesh, \\ Dhaka, Bangladesh
}

\begin{abstract}
The principle of this study is to minimize and categorize the large scale metadata to hasten an entity search in a wellorganized way. The objective is to addresses the fundamental issues of minimalist metadata visualization that make an entity identifiable by human assessment. It is very important to understand the dependency between an entity and the context of use of that entity to find the minimalist metadata visualization i.e., who is asking for what/whom at when and where. Hence, the study seeks to distinguish the need for requisite types, amount and order of metadata in different context. A survey result and user study on popular video sharing website is presented revealing interesting facts based on the common understanding and practice of metadata visualization. The results of our study will help to recognize the importance of the minimal attribute set to identify an entity. Finally, base on our findings, we proposed a solution which we believe can hasten any sort of entity search in an efficient way. ${ }^{1}$
\end{abstract}

\section{General Terms}

Data Visualization.

\section{Keywords}

Metadata, Minimalist Metadata Visualization, Entity, Context Boundary, Context Query.

\section{INTRODUCTION}

Metadata are data that defines higher level of data. Metadata is structured information that describes, explains, locates, or otherwise makes it easier to retrieve, use, or manage an information resource. Metadata is often called data about data or information about information [9]. For example what is the name of the data set? Who developed the data set? What geographic area does it cover? What themes of information does it include? How current are the data? Are there restrictions on accessing or using the data? The relation between data and metadata remains always the same from one level to another in the information hierarchy. Use of metadata is extremely important both in machine reasoning as well as for human inspection in large information space. Metadata is

\footnotetext{
${ }^{1}$ This work has been partially done during the PhD thesis research of Hasan $\mathrm{K}$. T. under the title "A User Centric Interface for the Management of Past, Present and Future Events" at the University of Trento, Italy in 2011[1].
}

capable of performing to describe what resources are and what they are about, and organizing those resources according to controllable criteria, allowing resources to be found by relevant criteria and providing digital identification and description for archiving and the preservation of resources.

Data describing data are often more ambiguous and/or confusing than the data itself and appears out of context. Data have been categorized, modeled and recorded by researchers and practitioners based on their metadata. This study treats entity for data and property for metadata. Set of properties being associated with each entity is not completely intended for human comprehension, rather only a part of it is to be visualized. There is a need for quick preview of entity properties. Another important aspect of the design is to determine the order of their arrangement i.e. setting the order of precedence of metadata visualization.

Minimalist metadata visualization for each entity type is subject to the context of use. Therefore, a user survey has been done to understand the need for required types, amount and order of metadata to find an entity in different context. The study took one entity type with five different contexts to illustrate how variant the user response in such understanding of requirements. There were 40 participants in the study seeking for a person in five different contexts given a set of properties (metadata) presented to them. An online survey tool has been used to perform the study. There are many fundamental questions we had to encounter: What are the metadata to be visualized to make an entity identifiable by human inspection? How much is sufficient? Or In which order?

Answering these questions is not a trivial task; therefore, no simple solution exists. For each entity, there exist numerous contexts where the answer varies. However, from the user survey this study came out with some very significant and exciting findings which specify that we really don't need much metadata to find an entity in a specific context. Afterward, a case study has been done on a popular video sharing website in different contexts where the target was to plot those findings. This work prepared five contexts and asked the user to search each of them in that website using a given set of keywords. These keywords are taken from frequently searched queries stored by Google engine. There were 50 participants in the study seeking for a specific video in five different contexts given a set of keywords (metadata) presented to them. Some key problems have been identified 
from the case study. For most of the scenarios, user failed to identity their desired video. Because some of the required metadata are not tagged in different contexts which failed the user to find preferred video result.

The study came out with some fundamental issues which will help to understand the importance of the minimal attribute set to identify an entity. Based on the key discovery, an equation with appropriate example has been proposed which may help to minimal the metadata set to find an entity efficiently.

\section{RELATED WORK}

Significant research has been done by the researcher from the past decays to minimize and categorize the large scale metadata. The Center for International Earth Science Information Network (CIESIN) at Columbia University is applying research on text visualization to the world of scientific data catalogs to increase efficiency in dealing with large metadata collections [3]. Vijay Kumar and Richard Furuta described a general framework for modeling and presenting temporal and other metadata [6]. Paul Mutton and Jennifer Golbeck worked on Visualization of Semantic Metadata and Ontologies, and revealed interesting information about the data relationships that can be extracted through visualization of the physical graph structure [7].

Another framework VisMeB is presented by Peter Klein and Frank Müller that support users to find relevant data and to enhance the possibilities of browsing and filtering an information space(e.g. digital library, web, geodata base, movie data base) [4]. Thomas Nocke and Heidrun Schumann proposed a way where they specified a variety of metadata supporting for visual mining tasks [5]. They developed an effective strategy for gathering metadata and included it in a general framework. Most of these research works provided the mechanism to formalize and categorize the large scale metadata. Some general frameworks have been introduced to model and present the metadata. On the other hand the goal of this study is to use the minimal set of metadata to find an entity by using the proposed equation.

\section{USER STUDY}

We have organized a study on user for 40 participants with one entity type for five different contexts which is aforementioned earlier. The intention was to depict how the user responses for required types, amount and sequence of metadata to find an entity in diverse contexts. There were total nine properties where eight were common while the ninth is clearly distinct. The participants had liberty to add properties of their own choice. Only one participant came up with an addition affiliation in few occurrences. They were -

\section{Picture of the person \\ 2. Full Name \\ 3. Nick name \\ 4. Current location \\ 5. Profession \\ 6. Date of birth \\ 7. Hometown \\ 8. Nationality}

And we will discuss about the ninth one in appropriate passage.

\subsection{The types and quantity of metadata}

We exploited five scenarios for five contexts and asked in a form of questions with predefined answers. The questions were as follows -

1. You are looking for your younger brother/sister (who happens to reside at the same home) on the web, what information is adequate to identify him/her from a search result? (Minimum context boundary)

2. You are looking for your favorite writer on the web, what are the information sufficient to find him/her from a search result? (Medium context boundary)

3. You are searching for your favorite actor/actress on the web, what are the information would you think ample to identify him/her from a list of people? (Medium context boundary)

4. You are searching for your childhood school friend after 15 years of detachment on the web, what are the information would you think mandatory to identify him/her? (High context boundary)

5. You are searching for the author of a prominent quote (you know it partially), what are the information would you think sufficient to identify $\mathrm{him} / \mathrm{her}$ from a list of people? (Higher context boundary)

What usually thought to be more or less close was deeply different from each other when users responded with their point of view. Here is the compendium table (Figure 1) with graph showing the result.

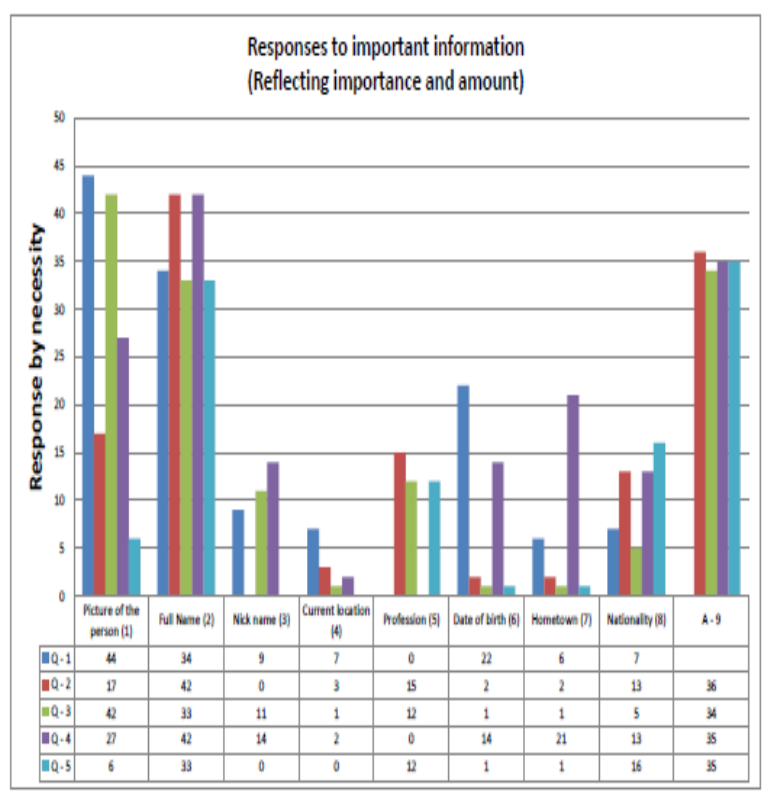

Fig 1: Responses to required metadata in five different contexts.

For question 1, picture, full name, date of birth ranked high respectively. Others being dwarfed can be considered less relevant or necessary. There was no requirement of ninth property for this question. If only three pieces of information are suffice for this context, and then we are probably very close to a minimalist view of entity properties.

The outcome is similar for the third context (question) where still the picture and the full name dominate with one addition of the ninth - "List of movies that s/he acted in". Note that another matter came to our concern about the size of the list. We are not obvious at this point how large the list could be since it may demand another study in its own right. 
Other than contexts one and four, people hardly cared for date of birth and this is surprisingly true for the fifth context that an image is not necessary.

For context four, full name, List of educational institution s/he attended (ninth property) and a picture appear to be adequate with the date of birth could help in some situations.

For question two, picture and profession followed by the full name and the list of writings (ninth property) and for five, the full name and the list of similar quotes (ninth property) are visualizing high in the graph.

Now if we have a quick look on the landscape, not much metadata is really needed given a specific context.

\subsection{The order of precedence}

When we are concerned at the order of precedence for the specific contexts, there is a tradeoff between the natural order and the user's choice. Our contemplation counted both for achieving correctness and completeness.

\section{Context 1: The person is very well known to the user}

A user looking for his/her brother/sister on the net provides the context of minimum context boundary. The picture tops all when we know the person with maximum possible details. Some participants ranked full name first to be ordered while others put it at the second position. No other metadata came at the first position. But we came across a decision that from the common paradigm of metadata visualization, we always put picture above the name label, should they come together because some persons are confound with label and photo sequence.

\section{Context 2: The person is known by his/her name and work}

Again the picture topped where an author (person) is mostly remembered by his/her name. Considering the order by picture first, then the list of literature and name at the last may appear little strange in reality. Therefore, again the picturename pair stays at the top followed by the list in order to maintain the convention as long as it does not conflict with the goal.

\section{Context 3: The person is known by his/her face and work}

While searching for our favorite actor/actress, the picture and the name pair alone formulates the entity identifiable. The anticipation did not work when participants put their opinion astonishingly. It's the name first followed by the picture and the list of the movies.

\section{Context 4: The person is remembered from childhood memories}

Twenty six participants ranked name at the top followed by the list of educational institution s/he attended and the name. Nick name also has significance to locate entity. Figure 2 interprets the consequence lucidly.

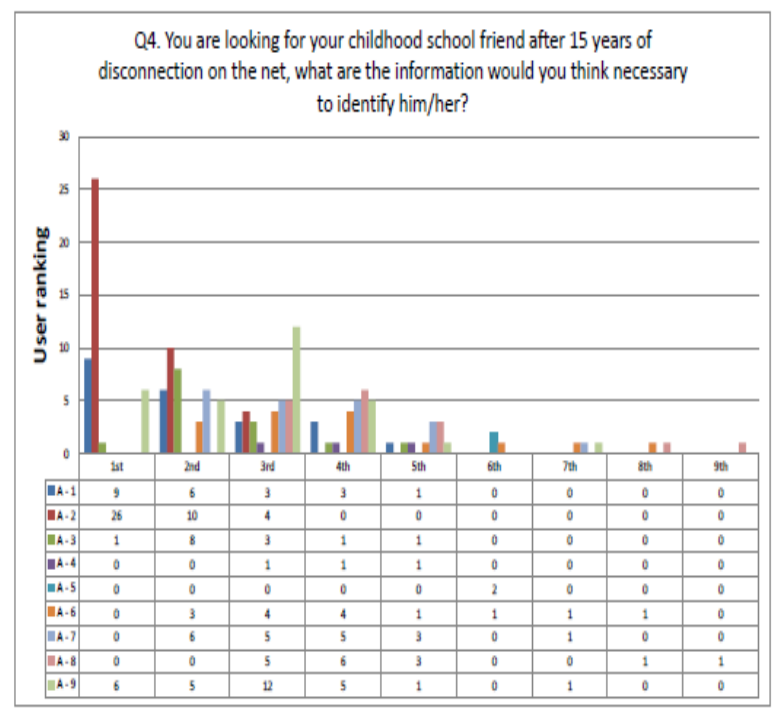

Fig 2: No matter what 15 years mean to make a big difference, people wanted to see the picture first.

Context 5: The person is not known, but a few of his/her words

One of the participants suggested to consider this context in a different way as the person is searched by the quote which is not prime properties of a person. Therefore, consider this totally a different context than the prior four. The person can only be identified by matching quotes as appears in the graph and can be learnt with rest of the metadata. The contest between similar quotes and name could have occurred due to the chances that the person is known, but not with relation to the given quotation.

For all five contexts, numerous opinions exist and for the values being smaller, we put them aside from further discussion. However, those less pertinent but ranked metadata are considered for extended view when necessary.

\section{CASE STUDY ON VIDEO-SHARING WEBSITE IN DIFFERENT CONTEXTS}

A video-sharing website is used to upload, view and share videos which is subsidiary of Google. When a user registers in such website, he /she provide the information like email, country, time zone, Date of Birth etc. The server of that website records the above information in Database. Now when a user uploads a video these information become the metadata of that video. This work tried to identify the application of required types, amount and order of metadata in a popular video-sharing website for different context. A video can be identified by following five criteria, though participants had the freedom to add properties of their own choice.

1. Title of the video

2. Thumb of the video

3. Upload Date

4. Number of Views

5. Uploaded Person

\subsection{The types and amount of metadata}

The experiment perceived five scenarios for five contexts and asked in a form of questions with predefined answers (mentioned in the previous section). The users are also given a set of keywords as mentioned earlier to search the video. The questions were as follows - 
1. To search the latest video footage of Hurricane in Bangladesh. Sample keyword: (hurricane in Bangladesh, hurricane in Bangladesh in most recent year etc.)

2. To search the last Euro cup football final video. Sample keyword: (last euro cup football final, current video of euro cup final etc.)

3. To search videos of live performance of Madonna last year. Sample keyword: (live performance of Madonna in last year etc.)

4. To search a video uploaded by user with the title "My son's first walk". Sample keyword: (my son's first walk on "uploaded date", my son's first walk by "username" etc.)

5. To search the latest episode of Big Bang Theory with sample keyword (latest episode of Big Bang Theory, recent update of Big Bang Theory etc.)

For most of the scenarios, user failed to identity their desired video but in some cases user recognized the videos by Title of the video and Upload Date. All of the users used our given keyword lists for searching though they had option for adding new query.

Confusion matrix has been used on the result of the survey to calculate the true positive rate for each of the given queries/keywords on each of the criteria in different contexts.

Now if we look at the results, some of the required metadata are not tagged in many contexts which failed the user to find preferred video result.

\subsection{Accuracy in different contexts}

Though the context query list was prepared from the frequently searched keywords and user had permission to add new queries, but in most of the cases user couldn't find their desired video in different contexts.

\section{Context 1: The event type and place is known.}

The user looked for the videos of hurricane in Bangladesh on our selected video sharing website provide the context of minimum context boundary.

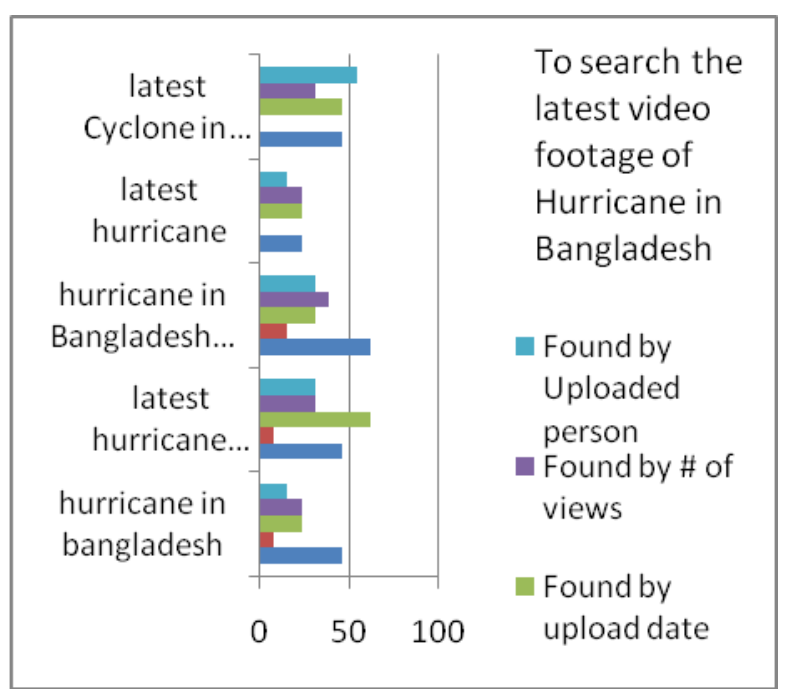

Fig 3: Some of the user recognized recent hurricane footage from the Thumb results though it's practically impossible.
The actual result of this search came up with series of videos containing "Hurricane", "Bangladesh" etc in its title or description though most of them are irrelevant and the result are not recent though we searched from most recent Hurricane which actually occurred in 2009 (Figure 7).

Context 2: The event and time boundary is known.

\begin{tabular}{|r|l|l|}
\hline $\begin{array}{r}\text { euro football } \\
\text { final } 2012\end{array}$ & $\begin{array}{l}\text { To search } \\
\text { the last } \\
\text { euro cup } \\
2012\end{array}$ & $\begin{array}{l}\text { Euro cup } \\
\text { football } \\
\text { video }\end{array}$ \\
$\begin{array}{r}\text { current video } \\
\text { of euro cup } \\
\text { latest euro } \\
\text { cup final }\end{array}$ & $\begin{array}{l}\text { Found by } \\
\text { Uploaded } \\
\text { person } \\
\text { last euro cup } \\
\text { football final }\end{array}$ & $\begin{array}{l}\text { Found by \# } \\
\text { of views }\end{array}$ \\
\hline
\end{tabular}

Fig 4: Pair of title and upload date shows comparatively high accuracy.

When the users searched for the last Euro cup football final video, some of the user recognized the video from title and the upload date. So the pair of title and upload date can identify this context though the accuracy is not satisfactory (Figure 8).

Context 3: The event type, entity and time boundary is known

While the user searched for videos of live performance of Madonna in last year, the results from different queries are totally irrelevant. For some of the keywords accuracy of thumb is higher but in other cases they are astonishingly low (Figure 9).

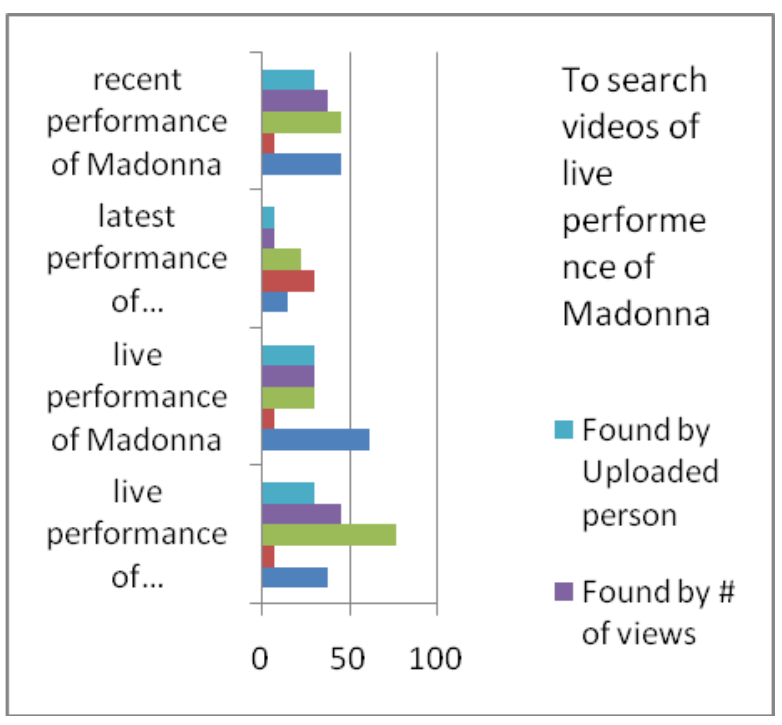

Fig 5: For context query 1, thumbs show higher accuracy but for other quires, results are scattered and not much accurate. 


\section{Context 4: The event title, entity and upload time.}

User looked for a video uploaded by the user few moments ago with the title for example "My son's first walk". For most of queries user couldn't find the video they had uploaded moments ago (Figure 10).

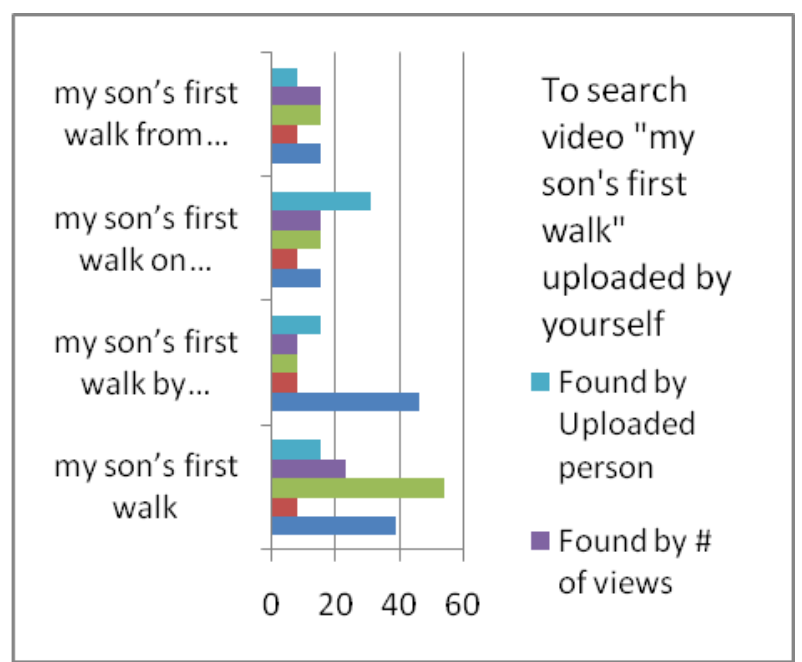

Fig 6: Most of the context queries showed very low correctness because most of the cases user failed to find desired video.

\section{Context 5: The event title and time edge is known.}

User tried to search the latest episode of a famous TV serial named "Big Bang Theory". Though they got results containing "latest" keyword in title but the episodes were actually not latest. Rather than considering upload date the search engine showed all the videos of "Big Bang Theory" that contains the keyword "latest" in title (Figure 11).

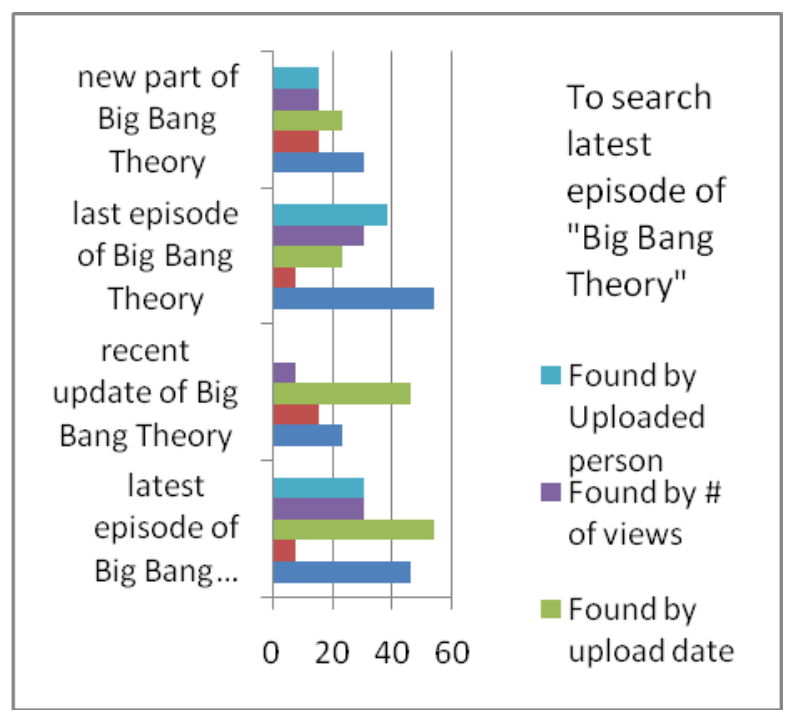

Fig 7: Though the entity and timeframe are known but results from different queries irrelevant.

\section{PROPOSED SOLUTION (CONTEXT BOUNDARY AND ITERATIVE QUERY)}

Now based on the survey and research, the study found out search results on selected popular video sharing website are not always precise and efficient and they can provide far better results based on the data they recorded from users. For all the cases, the common factor was, the search engine only has the capability to detect the words separately, rather than considering the minimal data set. Since, the engine isn't intelligent enough to understand the context of the query. Basically, context answers who, what, when and where [2] of the relevant entities. The question "who"; applies only to a context where people are explicitly associated. Furthermore, "which" is the question that essentially comes in play when an answer is incomplete or given in more general term. For instance, if an answer is "Joe is standing besides the car parking", then the obvious question may come to mind "which car parking?", if there are many around. If there is only one car parking area in the context and it is relatively large, then another "which" may come to place. The word "which"; is substitutive and replaced with the appropriate interrogation. This semantic gap can be sketched as a context boundary and the following illustration shows how recursively it runs till the desired result is found:

- Person X asks Person Y "where are you?"

- Person Y replies "At the car parking"

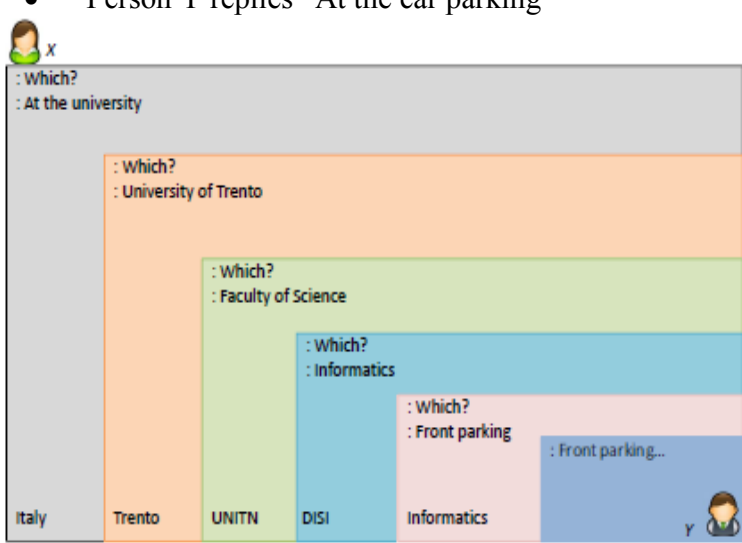

Fig 8: The less number of context boundary, the less semantic gap is.

If $\mathrm{X}$ were somewhere near to the $\mathrm{Y}$ 's position, then no more information would have been necessary to find Y. This context boundary is the essence of making metadata visualization decision.

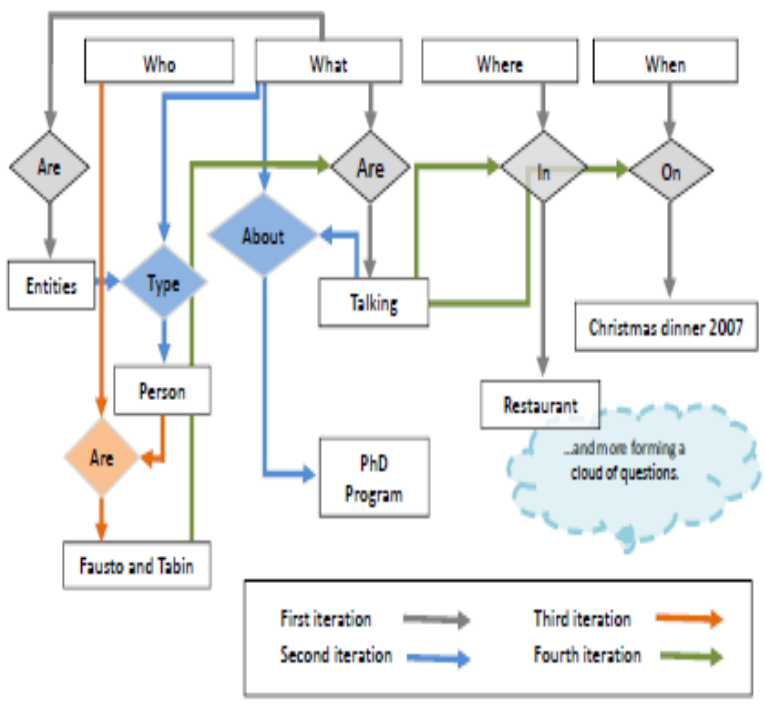

Fig 9: Questions are iterated demanding more answers if context boundary increases and goes on until a desired state is reached. 
A prior knowledge of the information seeker determines how much information would be necessary to find an answer. This is not however says in which order they are to be visualized (Figure 12).The definition of context, as plainly as it is seen, is not so plain. In many occasions, it may raise more questions than it answers like answering the questions to a child. If we consider a scenario where two persons are involved and want to see what exactly happening there, a finite number of inquiry (for known subject) or an infinite number of inquiry (for partially known subject) will arise.

However, describing to someone else far outside the context would result in a recursive query. Understanding the right context for both the user and the subject is required to provide some knowledge of -what information should come first, i.e. who is asking for what. At this point, the context answers "who" and "what". Answering these questions may open more new contexts. For instance, if the answer is "a person", then the obvious question may come to mind "who is/or the person?". From the figure 13, we can notice the answer came up with two persons "Fausto and Tabin". Here, "Fausto and Tabin" itself worked like a context and the next question came to the scenario is "What are they doing". The answer "talking" followed by with more queries, "What are they talking about?" and "where are they?" Answering these question move towards the question "when". Finally, after answering all the questions, the seeker finds the targeted entity.

After all that study has done, this work illustrates a formal definition of the context. Formally a context $C_{x}$ can be represented as:

$$
C_{x}=\{E, L, T\}
$$

Here, E stands for entity which can be either an object or an event. i.e. "Fausto and Tabin" is object type entity and "Christmas Dinner 2007" is an event type entity (figure 13).

Then, $\mathrm{L}$ represents the location where to search the object if performed entity is an object or it search the location where the event takes place contained in an event description. The location can be classified into two parts:

- Physical Location: The geographical place where the event takes place. A physical location is characterized by GPS coordinates, city and country specifications, etc. For example, the soccer match takes place at the Olympic Stadium of Rome.

- Logical Location: refers to a spatial domain concept (e.g., the match takes place in a stadium).

Finally, $\mathbf{T}$ stands for time association of an entity in different ways of capturing the aspect. The main ingredient that classifies time into two major groups in number.

- Relative Time (Chronostatic): This is a numberless reference to time. There is no date or quantitative measure for this class of time, e.g., yesterday, tomorrow, evening.

- Absolute Time (Chronometric): A quantitative measure of time expressed in numbers. Such time class follows relevant type of calendar. For us, this is a Gregorian calendar that is being used internationally for common usage.

From the example of the figure 13, we can observe that when we tried to search an entity in a certain context, the targeted entity itself may work like a context which may bring up with more possible contexts, until the desired result come. So, formally we can derive the context as:

$$
C_{T x}=\left\{C_{x}, L, T\right\}
$$

Applying appropriate set of minimal metadata can reduce the load to search an entity. If the context boundary is reduced with answering to a person already familiar with the scenario, it will end up soon with completeness.

\section{CONCLUSIONS AND FUTURE WORK}

This work has done a brief study to find out the essential issues of minimalist metadata visualization. Minimalist metadata visualization for entity recognition has been investigated through user studies and we came up with valuable findings and directions. Finally, an equation has been proposed which will help to reduce cognitive load to search an entity in a given context. As well as it will help to reduce the error and effort.

The future work have planned to develop a complete semantic engine that will understand the context boundary by collecting information about entity, context or event, try to reduce the context edge and minimize context metadata. The engine will have the ability to use iterative query if context boundary is unknown or too large.

\section{REFERENCES}

[1] Hasan K.T. A User Centric Interface for the Management of Past, Present and Future Events: International Doctorate School in Information and Communication Technology, DISI - University of Trento, April 2011

[2] Abowd G., Dey A., Brown P., Davies N., Smith M., and Steggles P. Towards a better understanding of context and Context-Awareness.In Hans-W Gellersen, editor, Handheld and Ubiquitous Computing, volume 1707 of Lecture Notes in Computer Science, chapter 29, pages 304-307. Springer Berlin / Heidelberg, Berlin, Heidelberg, November 1999.

[3] Mateescu I., Nowell L., Williams L., Moe K.L. Advanced visualization of scientific metadata. Center for International Earth Science Information Network, Columbia University.Support for this paper was provided by U.S. National Aeronautics and Space Administration under Contract NAS5-98162.

[4] Klein P., Mueller F., Reiterer H., Limbach T. Metadata Visualization with VisMeB. 7th International Conference on Information Visualisation 2003 (2003) Publisher: IEEE Computer Society, Pages: 600-605.

[5] Nocke T., Schumann H. Metadata for Visual Data Mining. Meta (2002) Volume: 2, Publisher: Citeseer.

[6] Kumar V., Furuta R., Allen R.B. Metadata Visualization for Digital Libraries: Interactive Timeline. Editing and Review. In DL '98: Proceedings of the third ACM conference on Digital libraries (1998), pp. 126-133.

[7] Mutton P. and Golbeck J. Visualization of Semantic Metadata and Ontologies: Proceedings of the Seventh International Conference on Information Visualization (IV’03) 1093-9547/03 \$17.00 @ 2003 IEEE. 
[8] Zeng M.L. and Qin J. Metadata: Published by NealSchuman Publishers, Inc. 100 William St., Suite 2004 New York, NY 10038.
[9] Understanding Metadata: NISO Press National Information Standards Organization 4733 Bethesda Avenue, Suite 300 Bethesda, MD 20814 USA Email: nisohq@niso.org URL: www.niso.org. 